\title{
3. Die Abteilung für Naturheilmedizin der Hufeland- Klinik, Bad Ems
}

ANDREAS A. BÜNZ

Die Abteilung für Naturheilmedizin an der Hufeland-Klinik in Bad Ems besteht bereits seit dem Jahre 2000 und hat sich seitdem als Zentrum für die stationäre naturheilkundliche und komplementär medizinische Versorgung der Patienten in Rheinland Pfalz und auch überregional etabliert. Speziell in Rheinland Pfalz wurde der Status der Naturheilverfahren im Krankenhaus politisch gestärkt, indem ein als eigenes, mit den klassischen Disziplinen wie Chirurgie oder Innere Medizin gleichgestelltes Fach mit der Bezeichnung Naturheilmedizin definiert und im Landesbettenplan festgeschrieben wurde. Die Abteilung verfügt über 40 Betten, die jeweils in Einzelzimmern untergebracht sind. Der Träger ist der katholische Orden der Schwestern der heiligen Maria Magdalena Postel. Ein Glücksgriff, denn christliche Werte und ganzheitliche Betrachtung des Menschen passen im Kontext integrativ erweiterter Medizin gut zusammen.

\subsection{Kostenübernahme}

Gesetzlich versicherten Patienten entstehen keine Kosten. Die Abteilung ist in dem Landesbettenplan aufgenommen und kann Patienten nach $\mathbb{3 9}$ SGB V vollstationär versorgen. Benötigt wird eine Krankenhauseinweisung durch den behandelnden Arzt. Üblicherweise wird die Indikation aber im Rahmen eines persönlichen Telefongespräches mit dem Patienten vor Aufnahme überprüft. Obwohl die Abteilung die Beihilfefähigkeit nach $\$ 7$ Abs. 4 BhV und $\mathbb{6} 6$ Abs. I Nr. 6 BhV hat, müssen beihilfeberechtigte Patienten vorab die Leistungszusage bei ihrer zuständigen Beihilfestelle einholen. Privat Versicherten wird vor Aufnahme 
geraten die schriftliche Zusage ihrer Krankenversicherung einzuholen. Aufgrund der hohen Nachfrage seitens der Patienten kann es zu Wartezeiten kommen.

\subsection{Indikationen}

Typischerweise werden Patienten mit folgenden Erkrankungen behandelt:

- Rheumatische Erkrankungen (Knochen- und Weichteilrheuma)

- Chronisch entzündliche Darmerkrankungen

- Entzündliche Hauterkrankungen

- Lungenerkrankungen und HNO - Erkrankungen mit entzündlicher oder allergischer Genese

- Begleitende Therapie bei Krebserkrankungen

- Chronische Schmerzzustände

- Fortgeschritten Lymphödeme

\subsection{Diagnostische Verfahren}

Zusammen mit der pneumologischen und der gastroenterologisch - geriatrischen Abteilung werden die gesamten diagnostischen Möglichkeiten der Inneren Medizin vorgehalten. Umfangreiche Kooperationen mit ärztlichen Partnern vor Ort vervollständigen die Diagnostik.

\subsection{Therapeutische Verfahren}

In der Abteilung werden Patienten gleichzeitig und gleichberechtigt mit moderner, Leitlinien - basierter Innerer Medizin und mit wissenschaftlich fundierten bzw. empirisch plausiblen komplementären Heilverfahren (Naturheilverfahren, Homöopathie, traditionelle chinesische Medizin) behandelt. Durch die Anwendung zahlreicher, unterschiedlicher Reize in hoher Frequenz und Intensität lassen sich auch bei chronischen Erkrankungen oft erhebliche Verbesserungen erzielen. Die typische Aufenthaltsdauer beträgt 13 bis 16 Tage.

Häufig angewendete komplementäre Verfahren sind

- Wasserheilkunde (Güsse, Bäder, Wickel)

- Wärmeanwendungen (z.B. Ganzkörperhyperthermie)

- Manuelle Therapien (z.B. Massagen, reflektorische Therapie)

- Krankengymnastik und Ergotherapie

- Ernährungstherapie und therapeutisches Heilfasten nach Buchinger

- Pflanzenheilkunde

- Ausleitende Verfahren (z.B. Blutegel, Schröpfkopfbehandlung) 
IV Portraits einzelner Kliniken und Abteilungen

- Neuraltherapie nach Huneke, lokale Mistelreiztherapie und Baunscheidt Verfahren

- Ordnungstherapie und Entspannungsverfahren

- Akupunktur

- Klassische Homöopathie

\subsection{Team}

In der Abteilung arbeiten ein interdisziplinäres therapeutisches Team von Fachärzten mit den Zusatzqualifikationen Naturheilverfahren, Homöopathie, TCM, speziell weitergebildetes Pflegepersonal, Physiotherapeuten, Masseure, Ergotherapeuten, Ernährungsberater und diplomierte Psychologen. 